02

\title{
Строение и колебательные спектры димерного комплексного фторида галлия (III) с катионом тетраметиламмония
}

\author{
() Е.И. Войт, Р.Л. Давидович, А.А. Удовенко, В.Б. Логвинова \\ Институт химии Дальневосточного отделения РАН, \\ 690022 Владивосток, Россия \\ e-mail: evoit@ich.dvo.ru
}

Поступила в редакцию 29.11.2018 г.

В окончательной редакции 13.06.2019 г.

Принята к публикации 20.06.2019 г.

\begin{abstract}
Определена кристаллическая структура димерного комплексного фторида галлия(III) $\left[\mathrm{N}\left(\mathrm{CH}_{3}\right)_{4}\right]_{2}\left[\mathrm{Ga}_{2} \mathrm{~F}_{8}\left(\mathrm{H}_{2} \mathrm{O}\right)_{2}\right]$, составленная из тетраэдрических катионов $\left[\mathrm{N}\left(\mathrm{CH}_{3}\right)_{4}\right]^{+}$и димерных комплексных анионов $\left[\mathrm{Ga}_{2} \mathrm{~F}_{8}\left(\mathrm{H}_{2} \mathrm{O}\right)_{2}\right]^{2-}$, образованных объединением двух слегка искаженных октаэдрических групп $\mathrm{GaF}_{5}\left(\mathrm{H}_{2} \mathrm{O}\right)$ общим ребром $\mathrm{F}-\mathrm{F}$. Водородными связями $\mathrm{O}-\mathrm{H} \cdots \mathrm{F}$ димерные комплексные анионы $\left[\mathrm{Ga}_{2} \mathrm{~F}_{8}\left(\mathrm{H}_{2} \mathrm{O}\right)_{2}\right]^{2-}$ объединяются в полимерные цепи, между которыми расположены катионы тетраметиламмония. На основании квантово-химических расчетов выполнено отнесение полос в колебательных спектрах синтезированного соединения. В спектрах идентифицированы полосы, относящиеся к участвующим в водородных связях колебаниям молекул $\mathrm{H}_{2} \mathrm{O}$, аниона и катиона.
\end{abstract}

Ключевые слова: галлий(III), комплексный фторид, кристаллическая структура, тетраметиламмоний, димер, колебательная спектроскопия.

DOI: 10.21883/OS.2019.12.48683.351-18

Комплексные фториды металлов с катионом тетраметиламмония (ТМА) проявляют сегнетоактивность [1], претерпевают фазовые переходы [2] и перспективны в качестве функциональных материалов. Сведения о комплексных фторидах $\mathrm{Ga}(\mathrm{III})$ с катионом TMA ограничены. Bukovec и Šiftar [3], исследуя систему $\mathrm{GaF}_{3}-\mathrm{N}\left(\mathrm{CH}_{3}\right)_{4} \mathrm{~F}-\mathrm{HF}-\mathrm{H}_{2} \mathrm{O}$ методом мольных отношений компонентов, установили, что при отношении $\mathrm{GaF}_{3}: \mathrm{N}\left(\mathrm{CH}_{3}\right)_{4} \mathrm{~F}$, равном $1: 3$, из раствора осаждается чистая твердая фаза, которая по результатам химического анализа соответствует формуле $\left[\mathrm{N}\left(\mathrm{CH}_{3}\right)_{4}\right] \mathrm{GaF}_{4} \cdot \mathrm{H}_{2} \mathrm{O}$. Соединение охарактеризовано межплоскостными расстояниями порошковой рентгенограммы, частотами ИК спектра, а также температурами дегидратации и термического разложения. Сведения о кристаллической структуре $\left[\mathrm{N}\left(\mathrm{CH}_{3}\right)_{4}\right] \mathrm{GaF}_{4} \cdot \mathrm{H}_{2} \mathrm{O}$ в литературе отсутствовали.

В продолжение проводимых систематических исследований комплексных фторидов индия(III) и галлия(III) [4,5] и с целью выявления новых структурных мотивов в этом классе соединений исследована кристаллическая структура $\left[\mathrm{N}\left(\mathrm{CH}_{3}\right)_{4}\right] \mathrm{GaF}_{4} \cdot \mathrm{H}_{2} \mathrm{O}$ в сочетании с детальным анализом колебательных спектров соединения.

\section{Экспериментальная часть}

\section{Синтез}

Соединение $\left[\mathrm{N}\left(\mathrm{CH}_{3}\right)_{4}\right] \mathrm{GaF}_{4} \cdot \mathrm{H}_{2} \mathrm{O}$ синтезировано препаративным методом путем взаимодействия гидрата окиси тетраметиламмония (25\%-раствор) и $\mathrm{GaF}_{3} \cdot 3 \mathrm{H}_{2} \mathrm{O}$ в водном растворе HF в интервале мольных отношений компонентов 2-4:1 с последующим изотермическим испарением раствора при комнатной температуре. Образовавшийся через несколько дней кристаллический осадок отделяли от маточного раствора фильтрованием под вакуумом, промывали небольшим количеством охлажденной воды и сушили на воздухе. Индивидуальность полученного соединения контролировали методами рентгенофазового анализа и ИК спектроскопии.

\section{Рентгеноструктурное исследование}

Рентгеновский эксперимент соединения выполнен на дифрактометре Bruker KAPPA APEX II (MoK $\alpha^{-}$ излучение, графитовый монохроматор). Сбор и редактирование данных, уточнение параметров элементарной ячейки проведены по программам SMART и SAINT Plus [6].

Структура исследованного соединения определена прямым методом и уточнена методом МНК по $F^{2}$ в анизотропном приближении для неводородоподобных атомов по программе SHELXTL/PC [7]. Атомы водорода определены из разностной электронной плотности и уточнены в изотропном приближении. Основные кристаллографические данные приведены в табл. 1. CIF-файл, содержащий полную информацию по исследованной структуре, был депонирован в CCDC под номером 1880819, откуда может быть свободно получен по запросу на интернет-сайте: www.ccdc.cam.ac.uk/data_request/cif. 


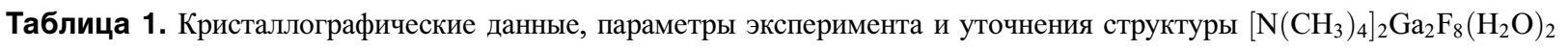

\begin{tabular}{|c|c|}
\hline Параметр & Значение \\
\hline 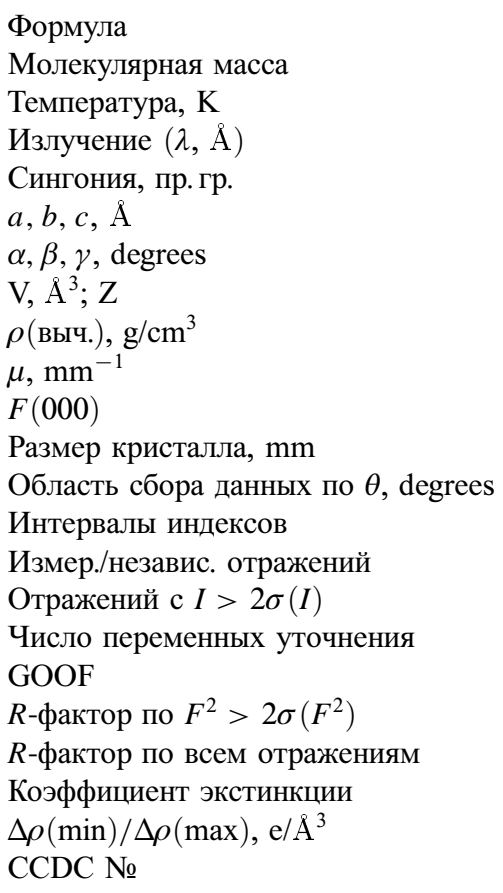 & $\begin{array}{l}\mathrm{C}_{8} \mathrm{H}_{28} \mathrm{~F}_{8} \mathrm{Ga}_{2} \mathrm{~N}_{2} \mathrm{O}_{2} \\
475.76 \\
296(2) \\
\text { МоK } K_{\alpha}(0.71073) \\
\text { Триклинная, } P \overline{1} \\
6.9232(1), 8.1853(2), 9.0931(2) \\
64.926(1), 82.866(1), 73.176(1) \\
446.75(2) ; 1 \\
1.768 \\
3.095 \\
240 \\
0.180 \times 0.180 \times 0.130 \\
2.473-39.387 \\
-11 \leq h \leq 12,-14 \leq k \leq 14,-16 \leq l \leq 16 \\
18756 / 5282[R(\text { int })=0.0221] \\
5282 \\
157 \\
1.006 \\
R_{1}=0.0228, w R_{2}=0.0544 \\
R_{1}=0.0316, w R_{2}=0.0572 \\
0.062(3) \\
-0.356 / 0.393 \\
1880819\end{array}$ \\
\hline
\end{tabular}

\section{Колебательная спектроскопия}

Инфракрасные спектры зарегистрированы на спектрометре Shimadzu IR Affinity-1 в диапазоне 400-4000 $\mathrm{cm}^{-1}$ с разрешением $4 \mathrm{~cm}^{-1}$ на окне KRS-5 с образцов, приготовленных в виде суспензии в вазелиновом и перфторированном маслах. Спектры комбинационного рассеяния (КР) кристаллических образцов записаны с разрешением $2 \mathrm{~cm}^{-1}$ на Bruker RFS100/S-спектрометре.

Квантово-химические расчеты проведены с применением программного комплекса Gamess [8]. Рассчитаны равновесная геометрия и частоты нормальных колебаний в гармоническом и ангармоническом (метод VSCF) приближениях. Вычисления выполнены на уровне теории функционала локальной плотности в сочетании с гибридным обменно-корреляционным потенциалом PBE0 $[9,10]$, хорошо зарекомендовавшим себя при описании водородных связей. Использованы электронно-коррелированные базисные наборы Даннинга: для $\mathrm{Ga}(\mathrm{III})$ (aug-cc-pVDZ-pp с остовным потенциалом), для атома H (d-aug-cc-pVDZ), для атомов F и O (aug-cc-pVDZ). Результаты получены с использованием оборудования ЦКП „Дальневосточный вычислительный

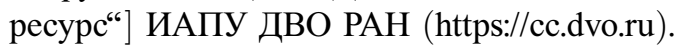

\section{Результаты и их обсуждение}

В исследованном интервале мольных отношений компонентов ТМАОН: $\mathrm{GaF}_{3} \cdot 3 \mathrm{H}_{2} \mathrm{O}$ 2-4:1 кристаллизуется одно соединение состава $\left[\mathrm{N}\left(\mathrm{CH}_{3}\right)_{4}\right] \mathrm{GaF}_{4} \cdot \mathrm{H}_{2} \mathrm{O}$.
Рентгенографическим исследованием установлено, что соединению $\left[\mathrm{N}\left(\mathrm{CH}_{3}\right)_{4}\right] \mathrm{GaF}_{4} \cdot \mathrm{H}_{2} \mathrm{O}$ соответствует состав $\quad\left[\mathrm{N}\left(\mathrm{CH}_{3}\right)_{4}\right]_{2}\left[\mathrm{Ga}_{2} \mathrm{~F}_{8}\left(\mathrm{H}_{2} \mathrm{O}\right)_{2}\right] . \quad$ Соединение $\left[\mathrm{N}\left(\mathrm{CH}_{3}\right)_{4}\right]_{2}\left[\mathrm{Ga}_{2} \mathrm{~F}_{8}\left(\mathrm{H}_{2} \mathrm{O}\right)_{2}\right]$ кристаллизуется в триклинной сингонии, пр.гр. $P \overline{1}$.

Структура $\left[\mathrm{N}\left(\mathrm{CH}_{3}\right)_{4}\right]_{2}\left[\mathrm{Ga}_{2} \mathrm{~F}_{8}\left(\mathrm{H}_{2} \mathrm{O}\right)_{2}\right]$ составлена из тетраэдрических катионов $\left[\mathrm{N}\left(\mathrm{CH}_{3}\right)_{4}\right]^{+}$и димерных комплексных анионов $\left[\mathrm{Ga}_{2} \mathrm{~F}_{8}\left(\mathrm{H}_{2} \mathrm{O}\right)_{2}\right]^{2-}$ (рис. 1, $a$ ), образованных объединением двух слегка искаженных октаэдрических групп $\mathrm{GaF}_{5}\left(\mathrm{H}_{2} \mathrm{O}\right)$ общим ребром $\mathrm{F}-\mathrm{F}$ (рис. $1, b$ ). В октаэдрических группах $\mathrm{GaF}_{5}\left(\mathrm{H}_{2} \mathrm{O}\right)$, образующих димерный комплекс, длины концевых связей $\mathrm{Ga}-\mathrm{F}$ равны $1.808(1)-1.865(1) \AA$. Мостиковые связи $\mathrm{Ga}-\mathrm{F}$ несколько длиннее и составляют $1.960(1) \AA$ и $1.981(1) \AA$. Координированная молекула $\mathrm{H}_{2} \mathrm{O}$ удалена от атома $\mathrm{Ga}$ на расстояние 2.022(1) $\AA$. В димерном комплексном анионе $\left[\mathrm{Ga}_{2} \mathrm{~F}_{8}\left(\mathrm{H}_{2} \mathrm{O}\right)_{2}\right]^{2-}$ координированные молекулы $\mathrm{H}_{2} \mathrm{O}$ занимают антиположения. Расстояние $\mathrm{Ga}(1)-\mathrm{Ga}(1)$ в димерном комплексном анионе составляет 3.081(2) А̊.

Координированная молекула $\mathrm{H}_{2} \mathrm{O}$ образует две водородные связи: одну внутримолекулярную $\mathrm{O}-\mathrm{H}(13) \cdots \mathrm{F}(3)$ с расстоянием 2.659(1) $\AA$ и вторую межмолекулярную $\mathrm{O}-\mathrm{H}(14) \cdots \mathrm{F}(2) \quad 2.568(1) \AA$, объединяющую димерные комплексные анионы $\left[\mathrm{Ga}_{2} \mathrm{~F}_{8}\left(\mathrm{H}_{2} \mathrm{O}\right)_{2}\right]^{2-}$ в полимерную цепь вдоль оси $a$ (рис. 1, b).

Катионы $\left[\mathrm{N}\left(\mathrm{CH}_{3}\right)_{4}\right]^{+}$имеют строение, близкое к правильному тетраэдру. Длины связей $\mathrm{N}-\mathrm{C}$ составляют 1.491(1)-1.494(1) $\AA$, а углы СNC изменяются от $109.18(9)^{\circ}$ до $109.88(9)^{\circ}$. Атомы углерода тетраметил- 


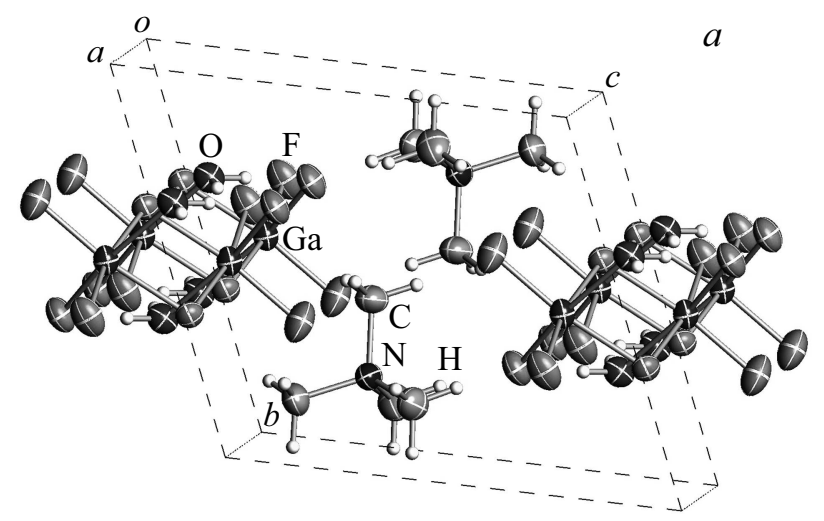

Рис. 1. Фрагменты кристаллической структуры $\left[\mathrm{N}\left(\mathrm{CH}_{3}\right)_{4}\right]_{2}\left[\mathrm{Ga}_{2} \mathrm{~F}_{8}\left(\mathrm{H}_{2} \mathrm{O}\right)_{2}\right] \quad(a)$ и объединение комплексных анионов $\left[\mathrm{Ga}_{2} \mathrm{~F}_{8}\left(\mathrm{H}_{2} \mathrm{O}\right)_{2}\right]^{2-}$ водородными связями в цепь $(b)$.

аммония образуют слабые водородные связи $\mathrm{C}-\mathrm{H} \cdots \mathrm{F}$ (3.280-3.430 $\AA$ ), которые объединяют анионные комплексы в решетке.

Экспериментальные колебательные спектры исследованного соединения $\left[\mathrm{N}\left(\mathrm{CH}_{3}\right)_{4}\right]_{2}\left[\mathrm{Ga}_{2} \mathrm{~F}_{8}\left(\mathrm{H}_{2} \mathrm{O}\right)_{2}\right]$ приведены на рис. 2. В ИК спектре соединения $\left[\mathrm{N}\left(\mathrm{CH}_{3}\right)_{4}\right]_{2}\left[\mathrm{Ga}_{2} \mathrm{~F}_{8}\left(\mathrm{H}_{2} \mathrm{O}\right)_{2}\right]$ в диапазоне $3500-2500 \mathrm{~cm}^{-1}$ наблюдается широкая интенсивная полоса с заметными максимумами $3300,3153,3066,3036 \mathrm{~cm}^{-1}$ и слабоинтенсивные полосы на низкочастотном крыле 2726, 2602, $2503 \mathrm{~cm}^{-1}$. Под этой полосой лежат колебания, соответствующие растяжениям связей $\mathrm{C}-\mathrm{H}$ катионов $\left(\mathrm{CH}_{3}\right)_{4} \mathrm{~N}^{+}$ и валентным колебаниям молекул $\mathrm{H}_{2} \mathrm{O}$, участвующих в водородных связях.

Внутренние колебания катиона $\left(\mathrm{CH}_{3}\right)_{4} \mathrm{~N}^{+}$тетраэдрической симметрии ранее рассмотрены и обсуждены в ряде работ $[11,12]$. Интерпретация характеристических полос катионов $\left(\mathrm{CH}_{3}\right)_{4} \mathrm{~N}^{+}$в спектрах соединения $\left[\mathrm{N}\left(\mathrm{CH}_{3}\right)_{4}\right]_{2}\left[\mathrm{Ga}_{2} \mathrm{~F}_{8}\left(\mathrm{H}_{2} \mathrm{O}\right)_{2}\right]$ выполнена, исходя из литературных данных. В спектре тетраэдрического $\left(\mathrm{CH}_{3}\right)_{4} \mathrm{~N}^{+}$, согласно неприводимому представлению $\Gamma_{\mathrm{Td}}=3 A 1+1 A 2+4 E+4 T 1+7 T 2$, моды симметрии $A 1, E$ и $T 2$ КР-активны, а ИК - только $T 2$.

Учитывая низкую симметрию положений катионов $\left(\mathrm{CH}_{3}\right)_{4} \mathrm{~N}^{+}$в кристаллической решетке и их искажение в результате взаимодействия с анионом, к валентным колебаниям $\mathrm{CH}_{3}$-групп катионов можно отнести интенсивные полосы с небольшой полушириной и максимумами в ИК $\sim 3066,3036 \mathrm{~cm}^{-1}$ и КР $\sim 3035,2978$, 2934 и $2837 \mathrm{~cm}^{-1}$ спектрах. Таким образом, происходит расщепление вырожденных мод и появление в спектре запрещенных переходов (табл. 2).

Деформационные колебания $\mathrm{CH}_{3}$-групп катионов проявляются в области $1500-1100 \mathrm{~cm}^{-1}$. Наиболее интенсивные полосы с максимумами $\sim 1498$ и $\sim 1471 \mathrm{~cm}^{-1}$ соответствуют плоскостным деформационным колебаниям $\mathrm{CH}_{3}$-групп, связанных с изменением $\mathrm{H}-\mathrm{C}-\mathrm{H}-$ углов. Внеплоскостные изгибные колебания $\mathrm{CH}_{3}$-групп имеют меньшую интенсивность, им в спектрах отвечают полосы $1426,1297,1185 \mathrm{~cm}^{-1}$.
Таблица 2. Положение полос поглощения катионов $\mathrm{N}\left(\mathrm{CH}_{3}\right)_{4}^{+}$ в спектрах $\left[\mathrm{N}\left(\mathrm{CH}_{3}\right)_{4}\right]_{2}\left[\mathrm{Ga}_{2} \mathrm{~F}_{8}\left(\mathrm{H}_{2} \mathrm{O}\right)_{2}\right]$ и их отнесение

\begin{tabular}{|c|c|c|}
\hline \multicolumn{2}{|c|}{$\begin{array}{c}\text { Экспериментальные частоты, } \\
\qquad \text { cm }^{-1}\end{array}$} & \multirow{2}{*}{$\begin{array}{c}\begin{array}{c}\text { Отнесение } \\
\text { (точечная группа) }\end{array} \\
{\left[\left(\mathrm{CH}_{3}\right)_{4} \mathrm{~N}\right]^{+}\left(T_{d}\right)}\end{array}$} \\
\hline иК & КР & \\
\hline $3066 \mathrm{c}$ & & $v_{13}(T 2) v_{a s} \mathrm{CH}_{3}$ \\
\hline \multirow{4}{*}{$\begin{array}{c}3036 \text { c } \\
2975-2830 \text { сл }\end{array}$} & 3035 oc & $v_{1}(A 1) v_{s} \mathrm{CH}_{3}$ \\
\hline & $2978 \mathrm{cp}$ & \\
\hline & $2934 \mathrm{cp}$ & $v_{5}(E) v_{a s} \mathrm{CH}_{3}$ \\
\hline & $2837 \mathrm{cp}$ & $v_{14}(T 2) v_{s} \mathrm{CH}_{3}$ \\
\hline \multirow[t]{4}{*}{$1498 \mathrm{c}$} & 1535 сл & \\
\hline & 1508 сл & $v_{15}(12) \delta \mathrm{CH}_{3}$ \\
\hline & $1471 \mathrm{oc}$ & $\nu_{6}(E) \delta \mathrm{CH}_{3}$ \\
\hline & 1426 сл & $v_{16}(T 2) \gamma \mathrm{CH}_{3}$ \\
\hline 1297 сл & 1297 сл & $v_{17}(T 2) \omega \mathrm{CH}_{3}$ \\
\hline \multirow[t]{2}{*}{1180 сл } & 1185 сл & $\nu_{7}(E) \omega \mathrm{CH}_{3}$ \\
\hline & & $v_{11}(T 1) \delta_{\mathrm{tw}} \mathrm{CH}_{3}$ \\
\hline \multirow[t]{4}{*}{$952 \mathrm{c}$} & $952 \mathrm{c}$ & $v_{18}(T 2) v_{a s} \mathrm{NC}_{4}$ \\
\hline & $753 c$ & $v_{3}(A 1) v_{s} \mathrm{NC}_{4}$ \\
\hline & 460 сл & $v_{19}(T 2) \delta \mathrm{NC}_{4}$ \\
\hline & $378 \mathrm{cp}$ & $\nu_{8}(E) \rho \mathrm{NC}_{4}$ \\
\hline
\end{tabular}

* Относительные интенсивности: ос - очень сильная, с - сильная, ср - средняя, сл - слабая.

Ниже по частоте расположены интенсивные узкие линии с максимумами $\sim 952$ (ИК, КР), 753, 460 и 378 (КР $\mathrm{cm}^{-1}$, которые являются характеристическими колебаниями группы $\mathrm{NC}_{4}$ катиона $\left(\mathrm{CH}_{3}\right)_{4} \mathrm{~N}^{+}$. Наиболее интенсивная в ИК и КР-спектрах полоса при $\sim 952 \mathrm{~cm}^{-1}$ соответствует асимметричным растяжениям связей N-C. Симметричный аналог этой полосы активен в КР и имеет максимум при $753 \mathrm{~cm}^{-1}$. Средней интенсивности в КР-спектре пики $\sim 460$ и $378 \mathrm{~cm}^{-1}$ соответствуют деформационным колебаниям $\mathrm{NC}_{4}$-групп.

$\mathrm{C}$ целью интерпретации полос в спектрах $\left[\mathrm{N}\left(\mathrm{CH}_{3}\right)_{4}\right]_{2}\left[\mathrm{Ga}_{2} \mathrm{~F}_{8}\left(\mathrm{H}_{2} \mathrm{O}\right)_{2}\right]$, соответствующих колебаниям комплексного аниона, проведен квантово-химический расчет колебательных частот димерной группы $\left[\mathrm{Ga}_{2} \mathrm{~F}_{8}\left(\mathrm{H}_{2} \mathrm{O}\right)_{2}\right]^{2-}$, составляющей основу исследованной 
Таблица 3. Экспериментальные и рассчитанные колебательные частоты и интенсивности комплексного аниона $\left[\mathrm{Ga}_{2} \mathrm{~F}_{8}\left(\mathrm{H}_{2} \mathrm{O}\right)_{2}\right]^{2-}$ $\left(\mathrm{S}_{2}\right)$ и их отнесение

\begin{tabular}{|c|c|c|c|c|c|c|}
\hline \multicolumn{2}{|c|}{$\begin{array}{l}\text { Экспериментальные } \\
\text { частоты, }{ }^{*} \mathrm{~cm}^{-1}\end{array}$} & \multirow{2}{*}{$\begin{array}{l}\text { Гармо- } \\
\text { ничные }\end{array}$} & \multirow{2}{*}{$\begin{array}{l}\text { Ангармо- } \\
\text { ничные }\end{array}$} & \multirow{2}{*}{$\begin{array}{c}\text { Интенсивность ИК/КР } \\
\left(D^{2} / \mu A^{2}\right) /\left(A^{4} / \mu\right)\end{array}$} & \multirow[t]{2}{*}{ Симметрия } & \multirow[t]{2}{*}{ Отнесение ${ }^{* *}$} \\
\hline ИК $\left(A_{u}\right)$ & $\mathrm{KP}\left(A_{g}\right)$ & & & & & \\
\hline $\begin{array}{c}3300 \text { пл ш } \\
3153 \text { ос ш } \\
1585 \text { ср ш } \\
1153 \text { сл } \\
842 \text { ср ш } \\
650 \text { ср ш }\end{array}$ & & $\begin{array}{l}3853 \\
3852 \\
3232 \\
3214 \\
1626 \\
1628 \\
1122 \\
1110 \\
811 \\
806 \\
445 \\
453\end{array}$ & $\begin{array}{l}3555 \\
3690 \\
3006 \\
2973 \\
1582 \\
1557 \\
1084 \\
1071 \\
772 \\
768 \\
599 \\
664\end{array}$ & $\begin{array}{c}3.5 / 0.0 \\
0.0 / 71.5 \\
41.9 / 0.0 \\
0.0 / 139.8 \\
2.5 / 0.0 \\
0.0 / 0.7 \\
0.0 / 1.6 \\
5.5 / 0.0 \\
7.1 / 0.0 \\
0.0 / 2.1 \\
0.0 / 0.8 \\
2.1 / 0.0\end{array}$ & $\begin{array}{l}A_{u} \\
A_{g} \\
A_{u} \\
A_{g} \\
A_{u} \\
A_{g} \\
A_{g} \\
A_{u} \\
A_{u} \\
A_{g} \\
A_{g} \\
A_{u}\end{array}$ & $\begin{array}{c}v \mathrm{O}-\mathrm{H} \\
v \mathrm{O}-\mathrm{H} \cdots \mathrm{F} \\
\delta \mathrm{H}_{2} \mathrm{O} \\
\omega \mathrm{H}_{2} \mathrm{O} \cdots \mathrm{F} \\
\omega \mathrm{H}_{2} \mathrm{O} \\
\rho \mathrm{H}_{2} \mathrm{O}\end{array}$ \\
\hline $\begin{array}{l}530 \mathrm{cp} \\
561 \mathrm{c} \\
478 \mathrm{c} \\
458 \text { сл }\end{array}$ & $\begin{array}{l}569 \text { с } \\
520 \text { c } \\
483 \text { сл } \\
460 \mathrm{cp}\end{array}$ & $\begin{array}{l}562 \\
532 \\
551 \\
532 \\
490 \\
482 \\
439 \\
428\end{array}$ & $\begin{array}{l}554 \\
536 \\
543 \\
537 \\
487 \\
501 \\
562 \\
486\end{array}$ & $\begin{array}{l}0.0 / 6.6 \\
8.1 / 0.0 \\
5.1 / 0.0 \\
0.0 / 1.0 \\
4.7 / 0.0 \\
0.0 / 7.9 \\
0.0 / 0.4 \\
3.7 / 0.0\end{array}$ & $\begin{array}{l}A_{g} \\
A_{u} \\
A_{u} \\
A_{g} \\
A_{u} \\
A_{g} \\
A_{g} \\
A_{u}\end{array}$ & $\begin{array}{c}v_{s} \mathrm{GaF}_{2}(\text { eq }) \\
v_{s} \mathrm{GaF}_{2}(\text { eq }) \\
v_{a s} \mathrm{GaF}_{2}(\mathrm{eq})+v_{a s} \mathrm{GaF}_{2}(\text { мост } \|) \\
v_{a s} \mathrm{GaF}_{2}(\mathrm{eq})+v_{a s} \mathrm{GaF}_{2}(\text { мост }=) \\
v \mathrm{GaF}_{2}(\mathrm{ax})+v_{s} \mathrm{GaF}_{2}(\text { мост }=)+\rho \mathrm{H}_{2} \mathrm{O} \\
\nu \mathrm{GaF}_{2}(\mathrm{ax})+v_{s} \mathrm{GaF}_{2}(\text { мост } \|)+\rho \mathrm{H}_{2} \mathrm{O} \\
v_{a s} \mathrm{GaF}(\mathrm{ax}) \mathrm{H}_{2} \mathrm{O}-v_{s} \mathrm{GaF}_{2}(\text { мост } \|) \\
v_{a s} \mathrm{GaF}(\mathrm{ax}) \mathrm{H}_{2} \mathrm{O}-v_{s} \mathrm{GaF}_{2}(\text { мост }=)\end{array}$ \\
\hline & 330 осл & $\begin{array}{l}346 \\
347 \\
328 \\
327\end{array}$ & $\begin{array}{l}360 \\
395 \\
328 \\
325\end{array}$ & $\begin{array}{l}3.1 / 0.0 \\
0.0 / 0.3 \\
0.0 / 0.2 \\
1.5 / 0.0\end{array}$ & $\begin{array}{c}A_{u} \\
A_{g} \\
A_{g} \\
A_{u}\end{array}$ & $\begin{array}{c}v_{s}, \delta(2 \mathrm{Fmocт})+\delta_{\omega}, \delta_{s c}\left(\mathrm{GaF}_{3} \mathrm{H}_{2} \mathrm{O}\right) \\
\left(\mathrm{H}_{2} \mathrm{O}, \text { ax, eq, мост }\right)\end{array}$ \\
\hline & $286 \mathrm{cp}$ & $\begin{array}{l}267 \\
243\end{array}$ & $\begin{array}{l}226 \\
228\end{array}$ & $\begin{array}{l}0.0 / 1.3 \\
0.6 / 0.0\end{array}$ & $\begin{array}{l}A_{g} \\
A_{u}\end{array}$ & $\begin{array}{c}v \mathrm{GaH}_{2} \mathrm{O}+v_{s}, \quad \delta(2 \text { Fмост }) \\
\left(\mathrm{H}_{2} \mathrm{O}, \text { мост }\right)\end{array}$ \\
\hline & $\begin{array}{l}258 \text { сл } \\
237 \text { сл } \\
199 \text { сл }\end{array}$ & $\begin{array}{l}297 \\
251 \\
232 \\
234 \\
201 \\
196\end{array}$ & $\begin{array}{l}288 \\
240 \\
266 \\
230 \\
258 \\
174\end{array}$ & $\begin{array}{l}2.5 / 0.0 \\
0.0 / 0.4 \\
1.7 / 0.0 \\
0.0 / 0.6 \\
0.0 / 0.8 \\
0.5 / 0.0\end{array}$ & $\begin{array}{l}A_{u} \\
A_{g} \\
A_{u} \\
A_{g} \\
A_{g} \\
A_{u}\end{array}$ & $\delta_{\omega}, \delta_{s c} \mathrm{GaF}_{3} \mathrm{H}_{2} \mathrm{O}\left(\right.$ eq.ax, $\left.\mathrm{H}_{2} \mathrm{O}\right)$ \\
\hline & $\begin{array}{l}143 \text { осл } \\
11 \text { осл }\end{array}$ & $\begin{array}{l}208 \\
171 \\
167 \\
154 \\
150 \\
112\end{array}$ & $\begin{array}{l}219 \\
180 \\
182 \\
177 \\
154 \\
112\end{array}$ & $\begin{array}{l}0.1 / 0.0 \\
0.0 / 0.6 \\
0.3 / 0.0 \\
0.0 / 0.2 \\
0.0 / 0.3 \\
0.0 / 0.1\end{array}$ & $\begin{array}{l}A_{u} \\
A_{g} \\
A_{u} \\
A_{g} \\
A_{g} \\
A_{g}\end{array}$ & $\rho, \delta_{t w} \mathrm{GaF}_{3} \mathrm{H}_{2} \mathrm{O}\left(\right.$ eq,ax, $\left.\mathrm{H}_{2} \mathrm{O}\right)$ \\
\hline & 186 сл & 186 & 172 & $0.0 / 0.4$ & $A_{g}$ & $v_{s}\left[\mathrm{GaF}_{3} \mathrm{H}_{2} \mathrm{O}+\mathrm{GaF}_{3} \mathrm{H}_{2} \mathrm{O}\right]$ \\
\hline & & $\begin{array}{r}136 \\
101 \\
86\end{array}$ & $\begin{array}{r}200 \\
101 \\
86\end{array}$ & $\begin{array}{l}0.1 / 0.0 \\
0.1 / 0.0 \\
0.1 / 0.0\end{array}$ & $\begin{array}{l}A_{u} \\
A_{u} \\
A_{u}\end{array}$ & $\delta\left[\mathrm{GaF}_{3} \mathrm{H}_{2} \mathrm{O}+\mathrm{GaF}_{3} \mathrm{H}_{2} \mathrm{O}\right]$ \\
\hline
\end{tabular}

* Относительные интенсивности: ос - очень сильная, с - сильная, ср - средняя, сл - слабая, осл - очень слабая, пл - плечо, ш - широкий. ** Обозначения колебаний: $v-$ валентные, деформационные: $\delta_{s c}-$ ножничные (в плоскости), $\omega-$ веерные $\left(\right.$ из плоскости), $\delta_{t} w-$ твист (из плоскости), $\rho$ - маятниковые (в плоскости). Колебаний мостиковых атомов F: поперек $(\|)$ и вдоль (=) направления мостиковых связей. 


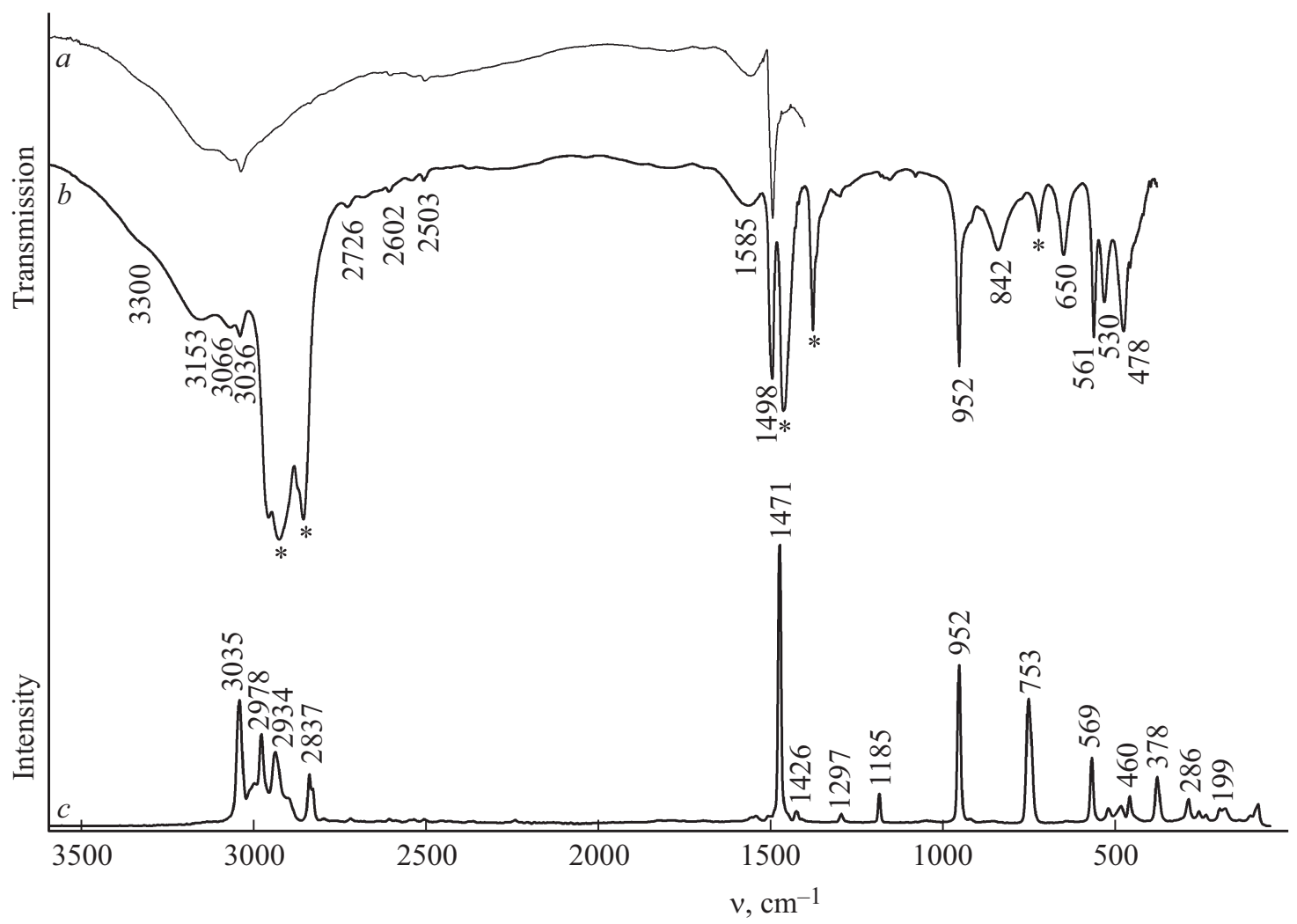

Рис. 2. ИК спектры $\left[\mathrm{N}\left(\mathrm{CH}_{3}\right)_{4}\right]_{2}\left[\mathrm{Ga}_{2} \mathrm{~F}_{8}\left(\mathrm{H}_{2} \mathrm{O}\right)_{2}\right] \quad(a)$ в перфторированном, $(b)$ в вазелиновом маслах; (c) КР-спектр $\left[\mathrm{N}\left(\mathrm{CH}_{3}\right)_{4}\right]_{2}\left[\mathrm{Ga}_{2} \mathrm{~F}_{8}\left(\mathrm{H}_{2} \mathrm{O}\right)_{2}\right]$. * - полосы вазелинового масла.
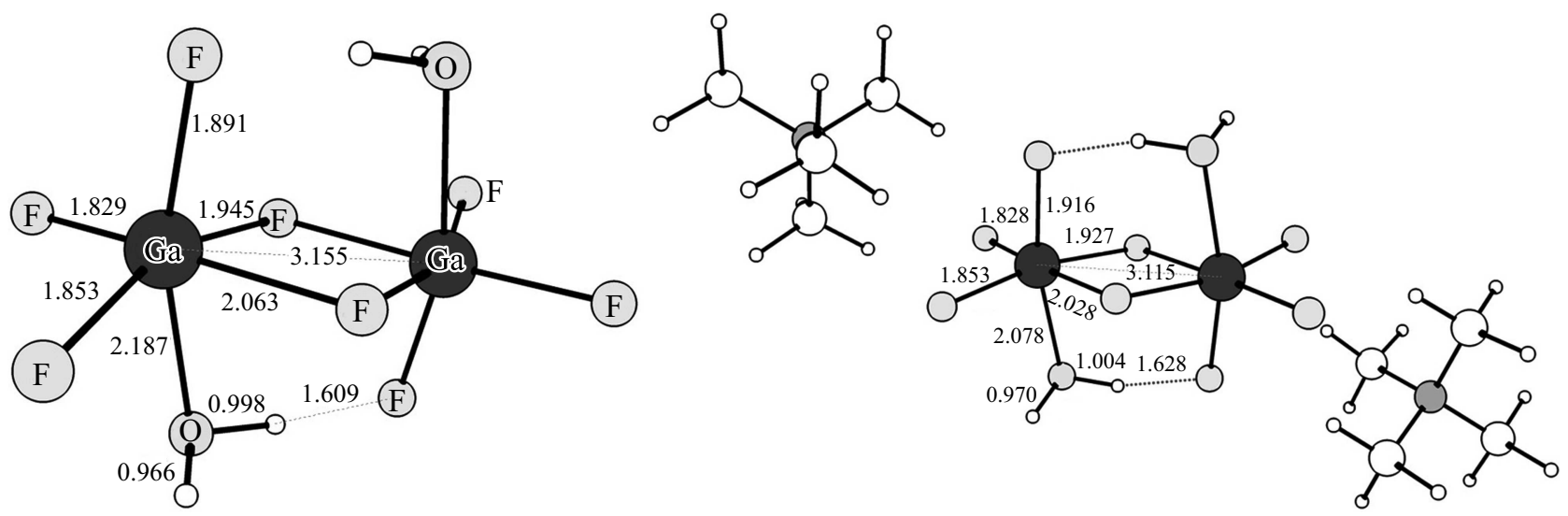

Рис. 3. Равновесная геометрия комплексного аниона $\left[\mathrm{Ga}_{2} \mathrm{~F}_{8}\left(\mathrm{H}_{2} \mathrm{O}\right)_{2}\right]^{2-}$ в отсутствие окружения и в окружении катионами.

структуры. Комплексный анион $\left[\mathrm{Ga}_{2} \mathrm{~F}_{8}\left(\mathrm{H}_{2} \mathrm{O}\right)_{2}\right]^{2-}$ имеет точечную симметрию $S_{2}$ и, согласно неприводимому представлению $\Gamma_{S 2}=21 A_{u}($ ИК $)+21 A_{g}(\mathrm{KP})$, его колебательный спектр характеризуется 42 фундаментальными колебательными частотами. Рассчитанная равновесная геометрия изолированного модельного аниона отличается от найденной в структуре $\left[\mathrm{N}\left(\mathrm{CH}_{3}\right)_{4}\right]_{2}\left[\mathrm{Ga}_{2} \mathrm{~F}_{8}\left(\mathrm{H}_{2} \mathrm{O}\right)_{2}\right]$, главным образом, завышением расстояния $\mathrm{Ga}-\mathrm{OH}_{2}$. Включение внешнесферного катионного окружения в расчет приводит к приближению равновесной геометрии к структурным данным (рис. 3).
Согласно результатам расчета (табл. 3), в экспериментальных колебательных спектрах $\left[\mathrm{N}\left(\mathrm{CH}_{3}\right)_{4}\right]_{2}\left[\mathrm{Ga}_{2} \mathrm{~F}_{8}\left(\mathrm{H}_{2} \mathrm{O}\right)_{2}\right] \quad$ к валентным колебаниям аниона можно отнести КР $\left(569,520,483,460 \mathrm{~cm}^{-1}\right)$ и ИК $\left(561,530,478,458 \mathrm{~cm}^{-1}\right)$ полосы. Первая пара частот $561 / 569 \mathrm{~cm}^{-1}($ ИК/КР) связана с растяжением экваториальных концевых связей $\mathrm{Ga}-\mathrm{F}$ аниона и относится к симметричной и асимметричной комбинациям валентных колебаний $v_{s} \mathrm{GaF}_{2}(\mathrm{eq})$. Вторая пара полос $530 / 520 \mathrm{~cm}^{-1} \quad($ ИК/КР $)$ соответствует комбинациям асимметричных валентных колебаний 
$v_{a s} \mathrm{GaF}_{2}(\mathrm{eq})$. Максимумы $478,458 \mathrm{~cm}^{-1}$ (ИК) и 483, $460 \mathrm{~cm}^{-1}$ (КР) можно отнести к комбинациям валентных колебаний аксиальных связей $\mathrm{Ga}-\mathrm{F}$ с небольшим вкладом валентных колебаний мостиковых связей $\mathrm{Ga}-\mathrm{F}-\mathrm{Ga}$.

В области 400-300 $\mathrm{cm}^{-1}$ лежат деформационные колебания димерного аниона с преимущественным участием мостиковых атомов фтора. Ниже $300 \mathrm{~cm}^{-1}$ находятся деформационные колебания, связанные с бо́льшим вкладом концевых атомов F (eq, ax), им в экспериментальном КР-спектре соответствуют полосы $\sim 286,258,237 \mathrm{~cm}^{-1}$ (табл. 3). В эту же группу вносят вклад валентные колебания $v \mathrm{Ga}-\mathrm{H}_{2} \mathrm{O}$.

Кроме того, в экспериментальном ИК спектре $\left[\mathrm{N}\left(\mathrm{CH}_{3}\right)_{4}\right]_{2}\left[\mathrm{Ga}_{2} \mathrm{~F}_{8}\left(\mathrm{H}_{2} \mathrm{O}\right)_{2}\right]$ можно выделить полосы, отвечающие внутренним колебаниям молекул $\mathrm{H}_{2} \mathrm{O}\left(C_{2 v}\right)$, координированных атомами $\mathrm{Ga}$ (рис. 2). К растяжениям связей $\mathrm{O}-\mathrm{H}$ в спектре можно отнести уширенный максимум $3153\left(v_{1}\right)$ и плечо при $3300\left(v_{3}\right) \mathrm{cm}^{-1}$. Форма и низкочастотное положение полосы валентных колебаний молекул $\mathrm{H}_{2} \mathrm{O}$ соответствуют образованию упрочненных водородных связей $\mathrm{O}-\mathrm{H} \cdots \mathrm{F}$, что согласуется с рентгеноструктурными данными (расстояния О-Н...F(3) $2.659 \AA$ и $\mathrm{O} \cdots \mathrm{F}(2) 2.568 \AA$ ) и результатами расчетов колебательных частот модельного аниона $\left[\mathrm{Ga}_{2} \mathrm{~F}_{8}\left(\mathrm{H}_{2} \mathrm{O}\right)_{2}\right]^{2-}$ (табл. 3), в котором одна из $\mathrm{O}-\mathrm{H}$-связей молекулы $\mathrm{H}_{2} \mathrm{O}$ образует внутридимерную водородную связь (рис. 3). Также проявление в ИК спектре полос либрационных колебаний молекул $\mathrm{H}_{2} \mathrm{O}\left(842\right.$ и $\left.650 \mathrm{~cm}^{-1}\right)$ с увеличенной полушириной подтверждает образование молекулами $\mathrm{H}_{2} \mathrm{O}$ в структуре упрочненных водородных связей.

Участие в колебаниях молекул $\mathrm{H}_{2} \mathrm{O}$ легкого атома водорода предполагает проявление эффектов ангармонизма [13]. Рассчитанные значения частот аниона $\left[\mathrm{Ga}_{2} \mathrm{~F}_{8}\left(\mathrm{H}_{2} \mathrm{O}\right)_{2}\right]^{2-}$ более точно передают положение в ИК спектре деформационных $\left(\delta \mathrm{H}_{2} \mathrm{O}\right)$ и либрационных колебаний молекул $\mathrm{H}_{2} \mathrm{O}$. Необычно низкое положение $\delta \mathrm{H}_{2} \mathrm{O}$ характерно также для соединений состава $\mathrm{MGaF}_{4} \cdot 2 \mathrm{H}_{2} \mathrm{O}$ $(\mathrm{M}=\mathrm{Rb}, \mathrm{Cs})[14]$.

\section{Заключение}

Синтезирован и структурно исследован новый комплексный фторид галлия $\left[\mathrm{N}\left(\mathrm{CH}_{3}\right)_{4}\right]_{2}\left[\mathrm{Ga}_{2} \mathrm{~F}_{8}\left(\mathrm{H}_{2} \mathrm{O}\right)_{2}\right]$. $\mathrm{B}$ кристаллической решетке атомы $\mathrm{Ga}$ образуют димерные комплексные анионы состава $\left[\mathrm{Ga}_{2} \mathrm{~F}_{8}\left(\mathrm{H}_{2} \mathrm{O}\right)_{2}\right]^{2-}$. Отличительной особенностью в организации кристаллической решетки $\left[\mathrm{N}\left(\mathrm{CH}_{3}\right)_{4}\right]_{2}\left[\mathrm{Ga}_{2} \mathrm{~F}_{8}\left(\mathrm{H}_{2} \mathrm{O}\right)_{2}\right]$ является система водородных связей. Координированная молекула $\mathrm{H}_{2} \mathrm{O}$ образует две водородные связи: одну внутримолекулярную О-Н(13)‥F(3) с расстоянием 2.659(1) $\AA$ и вторую межмолекулярную $\mathrm{O}-\mathrm{H}(14) \cdots \mathrm{F}(2)$ длиной 2.568(1) А, объединяющую димерные комплексные анионы $\left[\mathrm{Ga}_{2} \mathrm{~F}_{8}\left(\mathrm{H}_{2} \mathrm{O}\right)_{2}\right]^{2-}$ в полимерную цепь. Катионы тетраметиламмония компенсируют отрицательный заряд аниона и слабыми водородными связями $\mathrm{C}-\mathrm{H} \cdots \mathrm{F}$
(3.280-3.430 §) сшивают анионные цепи в каркас. Полученные экспериментальные колебательные (ИК, КР) спектры исследуемого вещества сравнены с результатами квантово-химических расчетов частот в гармоническом и ангармоническом приближениях. В спектрах идентифицированы полосы, относящиеся к участвующим в водородных связях колебаниям молекул $\mathrm{H}_{2} \mathrm{O}$, аниона и катиона.

\section{Список литературы}

[1] Göbel O.F., van Hummel G.J., Elshof J.E. // Z. Krystallogr. 2011. V. 226. P. 78. doi 10.1524/zkri.2011.1279

[2] Герасименко А.В., Гайворонская К.А., Давидович Р.Л., Диденко Н.А. // Журн. структурн. химии. 2016. Т. 57. C. 1226. doi 10.15372/JSC20160615

[3] Bukovec P., Šiftar J. // Monatsh. Chem. 1975. V. 106. P. 483. doi 10.1007/BF01150529

[4] Davidovich R.L., Fedorov P.P., Popov A.I. // Rev. Inorg. Chem. 2017. V. 37. P. 147. doi 10.1515/revic-2017-0010

[5] Давидович Р.Л., Удовенко А.А., Логвинова В.Б., Ткачев В.В., Шилов Г.В., Кайдалова Т.А. // Журн. струкутрн. химии. 2018. Т. 59. С. 1452. doi 10.26902/JSC20180621

[6] Bruker. APEX II. Bruker AXS Inc., Madison, Wisconsin, USA. 2008.

[7] Sheldrick G.M. // Acta Crystallogr. A. 2008. V. 64. P. 112. doi 10.1107/S0108767307043930

[8] Schmidt M.W., Baldridge K.K., Boatz J.A., Elbert S.T., Gordon M.S., Jensen J.H., Koseki S., Matsunaga N., Nguyen K.A., Su S.J., Windus T.L., Dupuis M., Montgomery J.A. // J. Comput. Chem. 1993. V. 14. P. 1347. doi.org/10.1002/jcc.540141112

[9] Perdew J.P., Burke K., Ernzerhof M. // Phys. Rev. Lett. 1996. V. 77. P. 3865. doi.org/10.1103/PhysRevLett.77.3865

[10] Adamo C., Barone V. // J. Chem. Phys. 1999. V. 110. P. 6158. doi $10.1063 / 1.478522$

[11] Wilson W.W., Christe K.O. // Inorg. Chem. 1989. V. 28. P. 4172. doi 10.1021/ic00321a027

[12] Malchus M., Jansen M. // Acta Crystallogr. B. 1998. V. 54. P. 494. https//doi.org/10.1107/S0108768197018351

[13] Bouman J.M. // J. Chem. Phys. 1978. V. 68. P. 608.

[14] Bukovec P., Orel B., Šiftar J. // Monatsh. Chem. 1973. V. 104. P. 194. https//doi.org/10.1007/BF00911160 